\title{
Utilização de dados parciais na seleção de codornas de corte para produção de ovos
}

\author{
Bruno Bastos Teixeira(1), Ricardo Frederico Euclydes ${ }^{(1)}$, Luciano Pinheiro da Silva(1), \\ Robledo de Almeida Torres(1), Felipe Gomes da Silva( ${ }^{(1)}$, Antonio Policarpo Souza Carneiro(1), \\ Helmut Gonçalves Lehner ${ }^{(1)}$ e Rafael Bastos Teixeira(1)
}

(1)Universidade Federal de Viçosa, Departamento de Zootecnia, Avenida Peter Henry Rolfs, s/no, CEP $36570-000$ Viçosa, MG. E-mail: brunobatzootecnia@yahoo.com.br, rbaja@ufv.br, lucianozooufv@yahoo.com.br, rtorres@ufv.br, felipepheu@yahoo.com.br, policarpo.carneiro@gmail.com, helmut_lehner@hotmail.com, rafaelzootecnia@yahoo.com.br

Resumo - O objetivo deste trabalho foi verificar a possibilidade de uso de dados parciais na seleção de codornas de corte para produção de ovos. Foram avaliados os grupos genéticos de codornas de corte UFV1 e UFV2, de origens distintas. Utilizaram-se informações de 1.632 matrizes, das quais 816 provieram do grupo genético UFV1, e 816 do grupo UFV2. Os parâmetros genéticos foram obtidos nos períodos parciais da $6^{\mathrm{a}}$ semana até a 24a $\left(\mathrm{P}_{24}\right)$, a 32a $\left(\mathrm{P}_{32}\right)$, a $40^{\mathrm{a}}\left(\mathrm{P}_{40}\right)$ e a $48^{\mathrm{a}}\left(\mathrm{P}_{48}\right)$ semanas, e no período total de produção de ovos $\left(\mathrm{P}_{52}\right)$, da $6^{\underline{a} \text { à }} 52^{\mathrm{a}}$ semana. Os componentes de variância e covariância e os parâmetros genéticos foram estimados pelo método da máxima verossimilhança restrita, pelo modelo animal unicaracterístico. A produção parcial e a total de ovos foram estimadas pelo modelo animal multicaracterístico, por meio do aplicativo Wombat. Para UFV1, os valores de herdabilidade foram: $0,09, \mathrm{P}_{24} ; 0,09, \mathrm{P}_{32} ; 0,09, \mathrm{P}_{40} ; 0,08, \mathrm{P}_{48} ; \mathrm{e} 0,07$ para $\mathrm{P}_{52} ;$ as correlações genéticas variaram de 0,79 a 0,99 . Para UFV2, os valores de herdabilidade foram: $0,09, \mathrm{P}_{24} ; 0,09, \mathrm{P}_{32} ; 0,10, \mathrm{P}_{40} ; 0,11$, $\mathrm{P}_{48} ;$ e 0,13 para $\mathrm{P}_{52}$; as correlações variaram de 0,70 a 0,99 . Para a seleção de UFV1, recomenda-se considerar a produção de ovos até a $40^{\underline{a}}$ semana e, para UFV2, até a $48^{\underline{a}}$ semana. As baixas estimativas de herdabilidade indicam que se devem fazer mudanças de manejo para controlar os efeitos de ambiente.

Termos para indexação: Coturnix coturnix, correlações genéticas, eficiência relativa, herdabilidade, seleção precoce, Wombat.

\section{Using partial data in the selection of meat-type quail for egg production}

\begin{abstract}
The objective of this work was to verify the possibility of using partial data in the selection of meat-type quail for egg production. The genetic groups UFV1 and UFV2 of meat-type quails, which are from different origins were evaluated. Information from 1,632 mothers, of which 816 from UFV1 and 816 from UFV2 genetic group, was used. The genetic parameters were obtained at the partial periods from the $6^{\text {th }}$ to $24^{\text {th }}\left(\mathrm{P}_{24}\right), 32^{\text {th }}\left(\mathrm{P}_{32}\right), 40^{\text {th }}\left(\mathrm{P}_{40}\right)$ and $48^{\text {th }}\left(\mathrm{P}_{48}\right)$ weeks, and at the total period of egg production $\left(\mathrm{P}_{52}\right)$, from the $6^{\text {th }}$ to $52^{\text {th }}$ week. The components of variance and covariance and the genetic parameters were estimated by restricted maximum likelihood, using a univariate animal model. The partial and total production of eggs were estimated by the multivariate animal model through the Wombat application. For UFV1, the heritability values were: $0.09, \mathrm{P}_{24} ; 0.09, \mathrm{P}_{32} ; 0.09, \mathrm{P}_{40} ; 0.08, \mathrm{P}_{48}$; and 0.07 for $\mathrm{P}_{52} ;$ and the genetic correlations ranged from 0.79 to 0.99 . For UFV2, the heritability values were: 0.09 for $\mathrm{P}_{24} ; 0.09, \mathrm{P}_{32} ; 0.10, \mathrm{P}_{40} ; 0.11, \mathrm{P}_{48} ;$ and 0.13 for $\mathrm{P}_{52}$; and correlations ranged from 0.70 to 0.99 . For UFV1 selection, it is recommended to consider egg production up to 40 weeks, and for UFV2 selection, egg production until the $48^{\text {th }}$ week. The low heritability estimates suggest that management changes should be done in order to control environmental effects.
\end{abstract}

Index Terms: Coturnix coturnix, genetic correlations, relative efficiency, heritability, early selection, Wombat.

\section{Introdução}

A coturnicultura brasileira vem crescendo de maneira expressiva em razão da possibilidade de rápido retorno do capital investido. Além disso, a qualidade da carne e dos ovos de codorna também tem aumentado (Teixeira et al., 2012).

A produção de ovos está entre as mais importantes características para a produção de codornas e pode influenciar diretamente o lucro da atividade de 
produção, que depende de três fatores principais: a idade ao primeiro ovo, taxa de postura e persistência da postura. A escolha do melhor critério de seleção para a produção de ovos deve levar em consideração esses três fatores (Winter et al., 2006; Rossi et al., 2010; Silva et al., 2013).

A seleção baseada no número de ovos produzidos no período total aumenta o intervalo de geração, e as aves são multiplicadas quando estão com baixa eficiência reprodutiva (Ribeiro et al., 2012; Teixeira et al., 2013). A determinação da produção de ovos em períodos parciais permite reduzir o intervalo de geração e possibilita a seleção de animais superiores mais cedo (Rossi et al., 2010). Assim, é possível aumentar a intensidade de seleção e reduzir os custos de produção, conforme se verifica em trabalhos com galinhas poedeiras (Al-Samarai et al., 2008; Venturini et al., 2012). No entanto, recomendações quanto a esses períodos parciais, para codornas de corte, não foram encontradas na literatura.

O objetivo deste trabalho foi verificar a possibilidade de uso de dados parciais na seleção de codornas de corte para produção de ovos.

\section{Material e Métodos}

Foram avaliados dois grupos genéticos, de origens distintas, de codorna de corte. Os grupos provieram do programa genético da UFV e são denominados UFV1 e UFV2. Utilizaram-se informações de 1.632 matrizes, das quais 816 do grupo genético UFV1 e 816 do grupo UFV2. Os dados foram coletados de 2007 a 2010, durante quatro gerações, nas quais foram acasalados, ao acaso, 204 matrizes com 102 machos.

Em cada geração, as aves foram alojadas em box de piso de concreto, na fase inicial de criação, forrado com cama de maravalha, equipado com círculo de proteção e aquecimento por meio de campânulas. Da fase inicial até o $42^{\circ}$ dia de vida, utilizou-se ração com $26 \%$ de proteína bruta e $2.950 \mathrm{kcal} \mathrm{kg}^{-1}$ de energia metabolizável, fornecida à vontade em comedouros tipo bandeja. O fornecimento de água também foi à vontade, em bebedouros tipo copo de pressão. Até o 14을 dia, foram utilizados programas de luz de 24 horas de iluminação. Do $14^{\circ}$ ao $28^{\circ}$ dia, adotou-se a iluminação natural. As seleções, baseadas em massa corporal, foram realizadas no 28 - dia de vida, quando foram selecionadas as 204 melhores fêmeas e os 102 melhores machos, dentro de cada grupo, em cada geração.

As codornas selecionadas foram transferidas para gaiolas galvanizadas individuais, para o controle da produção de ovos, conforme o critério de 1 macho para 2 fêmeas. A postura foi avaliada em gaiolas de $0,90 \mathrm{~m}$ de comprimento $\mathrm{x} 0,44 \mathrm{~m}$ de largura $\mathrm{x} 0,23 \mathrm{~m}$ de altura, em que $0,15 \mathrm{~m}$ da largura servia para aparador de ovos, com seis repartições.

A postura das fêmeas, em cada geração, foi avaliada pelo total de ovos colhidos da $6^{\mathrm{a}}$ à $52^{\mathrm{a}}$ semana, com um ano completo de postura avaliada. Foram avaliados os períodos parciais de produção de ovos da 6a à 24a $\left(\mathrm{P}_{24}\right)$, à $32^{\underline{a}}\left(\mathrm{P}_{32}\right)$, à $40^{\mathrm{a}}\left(\mathrm{P}_{40}\right)$ e à $48^{\mathrm{a}}\left(\mathrm{P}_{48}\right)$ semanas, e o período de produção total $\left(\mathrm{P}_{52}\right)$ da $6^{\underline{a}}$ à $52^{\underline{a}}$ semana. $\mathrm{O}$ programa $\mathrm{SAS}$, por meio do comando class, foi utilizado para auxiliar na escolha dos períodos parciais e na determinação do número de ovos de cada período.

Os componentes de variância e covariância e os parâmetros genéticos foram estimados pelo método da máxima verossimilhança restrita livre de derivadas (DFREML), com o uso do modelo animal multicaracterístico nos períodos de produção parcial e total, pelo programa Wombat (Meyer, 2007). Os valores iniciais, requisitados pelo Wombat, foram obtidos por meio das análises de variância e da literatura (Bonafé et al., 2011). Considerou-se o efeito fixo de geração/ eclosão para as características de postura. O modelo utilizado pode ser representado na forma matricial por $\mathrm{Y}=\mathrm{X} \beta+\mathrm{Z} \alpha+\varepsilon$, em que: $\mathrm{Y}$ é o vetor de observações; $X$ é a matriz de incidência de efeitos fixos; $\beta$ é o vetor de efeitos fixos; $Z$ é a matriz de incidência de efeitos aleatórios; $\alpha$ é o vetor de efeitos aleatórios; e $\varepsilon$ é o vetor de resíduos.

A eficiência relativa (ER) da seleção nos períodos parciais, em comparação à seleção pelo período total, foi calculada segundo a fórmula descrita por Falconer (1989): $E R=h_{p p} \times r_{\mathrm{g}} / h_{p t}$, em que: $h_{p p}$ é a raiz quadrada da herdabilidade do período parcial; $h_{p t}$ é a raiz quadrada da herdabilidade do período total; $r_{\mathrm{g}}$ é a correlação genética entre os períodos parcial e total. Utilizouse a eficiência relativa, em razão da possibilidade de comparar o ganho genético em uma característica, por meio da resposta correlacionada em outra. Se a razão for maior que a unidade, a resposta correlacionada é favorável, e o ganho genético será maior que a seleção direta na outra característica. 


\section{Resultados e Discussão}

Nos grupos genéticos UFV1 e UFV2, a produção de ovos avaliada durante o período total de produção $\left(\mathrm{P}_{52}\right)$ apresentou a maior variação (Tabela 1). Os coeficientes de variação ficaram próximos ao valor de 16,54\% encontrado por Ribeiro et al. (2012). Essa maior variação pode ser explicada pelo fato de este período abranger o fim do período de postura, que apresenta baixa eficiência reprodutiva, o que leva a uma maior variação na quantidade de ovos produzidos pelas codornas (Bonafé et al., 2011).

As estimativas de herdabilidade encontradas para os grupos genéticos UFV1 e UFV2 foram de baixa magnitude e variaram de 0,07 a 0,09 e de 0,09 a 0,13 (Tabela 2). Em relação ao grupo genético UFV1, Ribeiro et al. (2012), ao trabalhar com o mesmo grupo, encontraram estimativas semelhantes de herdabilidade, que variaram de 0,03 a 0,16 . Venturini et al. (2012), em estudos com galinhas poedeiras, também encontraram estimativas semelhantes de herdabilidade, que variaram de 0,09 a 0,27 e 0,03 a 0,33. A característica produção de ovos, normalmente, apresenta valores de herdabilidade de baixos a moderados, que indicam que grande parte da variação da característica decorre de variações no ambiente (Teixeira et al., 2012).

Para o grupo genético UFV2, as estimativas de herdabilidade foram semelhantes às obtidas por Teixeira et al. (2012) e Venturini et al. (2012), porém inferiores às encontradas por Winter et al. (2006)

Tabela 1. Produção de ovos, desvios padrão (DP), coeficientes de variação $(\mathrm{CV})$ e erro-padrão das médias $(\mathrm{EP})$ de produção de ovos, para os grupos genéticos UFV1 e UFV2.

\begin{tabular}{lcccc}
\hline Período $^{(1)}$ & Média & DP & CV $(\%)$ & EP \\
\hline $\mathrm{P}_{24}$ & 110,16 & 16,23 & 14,73 & 0,63 \\
$\mathrm{P}_{32}$ & 158,37 & 22,36 & 14,11 & 0,88 \\
$\mathrm{P}_{40}$ & 202,33 & 30,80 & 15,22 & 1,22 \\
$\mathrm{P}_{48}$ & 245,69 & 41,32 & 16,81 & 1,65 \\
$\mathrm{P}_{52}$ & 267,20 & 47,33 & 17,71 & 1,90 \\
\hline & & \multicolumn{3}{c}{ UFV2 } \\
$\mathrm{P}_{24}$ & 107,78 & 18,37 & 17,04 & 0,72 \\
$\mathrm{P}_{32}$ & 153,64 & 27,63 & 17,98 & 1,09 \\
$\mathrm{P}_{40}$ & 194,02 & 39,24 & 20,22 & 1,44 \\
$\mathrm{P}_{48}$ & 233,11 & 52,77 & 22,63 & 2,11 \\
$\mathrm{P}_{52}$ & 251,50 & 59,85 & 23,39 & 2,42 \\
\hline
\end{tabular}

${ }^{(1)} \mathrm{P}_{24}$, produção de ovos no período parcial até a 24늘 semana; $\mathrm{P}_{32}$, até a $32^{\text {a }}$ semana; $\mathrm{P}_{40}$, até a $40^{\text {a }}$ semana; $\mathrm{P}_{48}$, até a $48^{\text {a }}$ semana; e $\mathrm{P}_{52}$, período total até a $52^{2}$ semana. e Paiva et al. (2010). Em trabalho com o mesmo grupo genético, Ribeiro et al. (2012) encontraram estimativas que variaram de 0,20 a 0,25 para todos os períodos estudados. Portanto, as baixas estimativas de herdabilidade encontradas para os grupos UFV1 e UFV2 indicam alta variabilidade devida ao ambiente entre as codornas. Entre os fatores ambientais relacionados à produção de ovos estão: época de nascimento, nutrição, programa de luz, massa corporal etc.

As maiores estimativas de correlação genética em UFV1 foram observadas entre os períodos $\mathrm{P}_{40} \times \mathrm{P}_{52}$, e entre $\mathrm{P}_{48} \times \mathrm{P}_{52}$ (Tabela 3 ). Os valores observados $(0,79$ a 0,99$)$ foram semelhantes aos encontrados por Winter et al. (2006) em codornas japonesas $(0,71$ a 0,99), e aos encontrados por Paiva et al. (2010) sobre galinhas poedeiras $(0,67$ a 0,94$)$. Portanto, os dados desses períodos poderiam ser utilizados para seleção precoce. No entanto, ao analisar ambos os períodos, a seleção baseada nos dados de $\mathrm{P}_{40}$ apresentou um maior valor de eficiência relativa; dessa forma, esse período é o mais recomendável para a seleção das codornas de corte, para produção de ovos. Assim, o grupo genético UFV1 pode se beneficiar da redução do intervalo de geração, o que possibilitaria um maior ganho genético por unidade de tempo.

Para UFV2, as estimativas de correlação genética variaram de 0,70 a 0,99 (Tabela 3). Esses valores são semelhantes aos encontrados por Winter et al. (2006) e Paiva et al. (2010) e superiores ao encontrados por Ribeiro et al. (2012) e Venturini et al. (2012). Essas

Tabela 2. Estimativas de herdabilidade $\left(\mathrm{h}^{2}\right)$ e das variâncias genética aditiva $\left(\sigma_{\mathrm{a}}^{2}\right)$, ambiental $\left(\sigma_{\mathrm{e}}^{2}\right)$ e fenotípica $\left(\sigma_{\mathrm{p}}^{2}\right)$ da produção de ovos, nos grupos genéticos UFV1 e UFV2.

\begin{tabular}{|c|c|c|c|c|}
\hline Período $^{(1)}$ & $\mathrm{h}^{2}$ & $\sigma_{a}^{2}$ & $\sigma_{e}^{2}$ & $\sigma_{p}^{2}$ \\
\hline & \multicolumn{4}{|c|}{ UFV1 } \\
\hline $\mathrm{P}_{24}$ & 0,09 & 24,35 & 238,97 & 263,32 \\
\hline $\mathrm{P}_{32}$ & 0,09 & 47,06 & 441,50 & 484,56 \\
\hline $\mathrm{P}_{40}$ & 0,09 & 80,21 & 765,77 & 845,98 \\
\hline $\mathrm{P}_{48}$ & 0,08 & 121,04 & $1.288,60$ & $1.409,60$ \\
\hline \multirow[t]{2}{*}{$\mathrm{P}_{52}$} & 0,07 & 132,18 & $1.688,50$ & $1.820,20$ \\
\hline & \multicolumn{4}{|c|}{ UFV2 } \\
\hline $\mathrm{P}_{24}$ & 0,09 & 30,00 & 303,61 & 333,62 \\
\hline $\mathrm{P}_{32}$ & 0,09 & 68,94 & 674,94 & 743,89 \\
\hline $\mathrm{P}_{40}$ & 0,10 & 141,43 & $1.263,50$ & $1.404,90$ \\
\hline $\mathrm{P}_{48}$ & 0,11 & 276,73 & $2.099,00$ & $2.375,80$ \\
\hline $\mathrm{P}_{52}$ & 0,13 & 391,01 & $2.589,50$ & $2.980,60$ \\
\hline
\end{tabular}

${ }^{(1)} \mathrm{P}_{24}$, produção de ovos no período parcial até a 24늘 semana; $\mathrm{P}_{32}$, até a 32a semana; $\mathrm{P}_{40}$, até a $40^{\mathrm{a}}$ semana; $\mathrm{P}_{48}$, até a $48^{\mathrm{a}}$ semana; e $\mathrm{P}_{52}$, período total até a 52 a semana. 
Tabela 3. Estimativas das correlações genética $\left(\mathrm{r}_{\mathrm{g}}\right)$, ambiental $\left(\mathrm{r}_{\mathrm{e}}\right)$ e fenotípica $\left(\mathrm{r}_{\mathrm{p}}\right)$, de cada período parcial $\left(\mathrm{P}_{24}\right.$, $\left.\mathrm{P}_{32}, \mathrm{P}_{40}, \mathrm{P}_{48}\right)$ com o período total $\left(\mathrm{P}_{52}\right)$, e eficiência relativa da seleção, para os grupos genéticos UFV1 e UFV2.

\begin{tabular}{lcccc}
\hline Período & $\mathrm{r}_{\mathrm{g}}$ & $\mathrm{r}_{\mathrm{e}}$ & $\mathrm{r}_{\mathrm{p}}$ & Eficiência relativa \\
\hline $\mathrm{P}_{24} \times \mathrm{P}_{52}$ & 0,79 & 0,522 & 0,544 & 0,90 \\
$\mathrm{P}_{32} \times \mathrm{P}_{52}$ & 0,89 & 0,738 & 0,751 & 1,03 \\
$\mathrm{P}_{40} \times \mathrm{P}_{52}$ & 0,99 & 0,893 & 0,901 & 1,14 \\
$\mathrm{P}_{48} \times \mathrm{P}_{52}$ & 0,99 & 0,985 & 0,986 & 1,09 \\
\hline & \multicolumn{3}{c}{$\mathrm{UFV} 2$} \\
$\mathrm{P}_{24} \times \mathrm{P}_{52}$ & 0,70 & 0,570 & 0,584 & 0,59 \\
$\mathrm{P}_{32} \times \mathrm{P}_{52}$ & 0,88 & 0,804 & 0,812 & 0,75 \\
$\mathrm{P}_{40} \times \mathrm{P}_{52}$ & 0,94 & 0,926 & 0,928 & 0,83 \\
$\mathrm{P}_{48} \times \mathrm{P}_{52}$ & 0,99 & 0,989 & 0,989 & 0,94
\end{tabular}

${ }^{(1)} \mathrm{P}_{24}$, produção de ovos no período parcial até a $24^{\mathrm{a}}$ semana; $\mathrm{P}_{32}$, até a $32^{\mathrm{a}}$ semana; $\mathrm{P}_{40}$, até a $40^{\mathrm{a}}$ semana; $\mathrm{P}_{48}$, até a $48^{\mathrm{a}}$ semana; e $\mathrm{P}_{52}$, período total até a $52^{\mathrm{a}}$ semana.

correlações, aliadas aos valores de eficiência relativa, indicam que a adoção dos dados dos períodos $\mathrm{P}_{24} \mathrm{e}$ $\mathrm{P}_{32}$, para a seleção, traria menor ganho que seleção baseada na produção total. Contudo, o uso de dados de produção total acarreta maior intervalo de geração. Portanto, entre os períodos de produção parcial, o $\mathrm{P}_{48}$ seria o mais indicado para realizar a seleção no grupo UFV2, visto que apresenta maior estimativa de herdabilidade, alto valor de correlação genética com a produção total e maior valor de eficiência relativa. Neste caso, o aumento no número de gerações poderia aumentar o ganho genético com o passar do tempo.

\section{Conclusões}

1. A seleção baseada em períodos de produção parcial de ovos é viável.

2. Para o grupo genético UFV1, recomenda-se a seleção precoce com os dados relativos até a $40^{\mathrm{a}}$ semana de vida e, para UFV2, até a $48^{\text {a }}$ semana.

3. As baixas estimativas de herdabilidade indicam que se devem fazer mudanças de manejo para o controle dos efeitos de ambiente, para obtenção de menor variabilidade dentro dos grupos genéticos.

\section{Agradecimento}

À Coordenação de Aperfeiçoamento de Pessoal de Nível Superior (Capes), ao Conselho Nacional de Desenvolvimento Científico e Tecnológico (CNPq) e à Fundação de Amparo à Pesquisa do Estado de Minas Gerais (Fapemig), pelo apoio financeiro.

\section{Referências}

AL-SAMARAI, F.R.; AL-KASSIE, G.A.; AL-NEDAWI, A.M.; AL-SOUDI, K.A.-A. Prediction of total egg production from partial or cumulative egg production in a stock of white leghorn hens in Iraq. International Journal of Poultry Science, v.7, p.890-893, 2008. DOI: 10.3923/ijps.2008.890.893.

BONAFÉ, C.M.; TORRES, R. de A.; SARMENTO, J.L.R.; SILVA, L.P. da; RIBEIRO, J.C.; TEIXEIRA, R.B.; SILVA, F.G. da; SOUSA, M.F. Modelos de regressão aleatória para descrição da curva de crescimento de codornas de corte. Revista Brasileira de Zootecnia, v.40, p.765-771, 2011. DOI: 10.1590/S151635982011000400009 .

FALCONER, D.S. Introduction to quantitative genetics. $3^{\text {rd }}$ ed. Harlow: Longman, 1989. 428p.

MEYER, K. WOMBAT - a tool for mixed model analyses in quantitative genetics by restricted maximum likelihood (REML). Journal of Zhejiang University Science B, v.8, p.815-821, 2007. DOI: 10.1631/jzus.2007.B0815.

PAIVA, A.L. da C.; TEIXEIRA, R.B.; YAMAKI, M.; MENEZES, G.R. de O.; LEITE, C.D.S.; TORRES, R. de A. Análise de componentes principais em características de produção de aves de postura. Revista Brasileira de Zootecnia, v.39, p.285-288, 2010. DOI: $10.1590 / \mathrm{S} 1516-35982010000200009$.

RIBEIRO, J.C.; SILVA, L.P. da; SOUSA, M.F.; LEITE, C.D.S.; BONAFÉ, C.M.; CAETANO, G. da C.; CRISPIM, A.C.; TORRES, R. de A. Genetic evaluation for egg mass in partial periods and complete period in meat quails. Revista Brasileira de Zootecnia, v.41, p.1158-1162, 2012. DOI: 10.1590/S151635982012000500012 .

ROSSI, R.M.; MARTINS, E.N. Influência das coletas sistemáticas e parciais na seleção de codornas por meio de curvas de probabilidade de postura. Revista Brasileira de Zootecnia, v.39, p.1699-1707, 2010. DOI: 10.1590/S1516-35982010000800011.

SILVA, L.P. da; RIBEIRO, J.C.; CRISPIM, A.C.; SILVA, F.G. da; BONAFÉ, C.M.; SILVA, F.F.; TORRES, R. de A. Genetic parameters of body weight and egg traits in meat-type quail. Livestock Science, v.153, p.27-32, 2013. DOI: 10.1016/j. livsci.2013.01.014.

TEIXEIRA, B.B.; EUCLYDES, R.B.; TEIXEIRA, R.B.; SILVA, L.P. da; TORRES, R. de A.; SILVA, F.G. da; LEHNER, H.G.; CAETANO, G. da C. Herdabilidade de características de produção e postura em matrizes de codornas de corte. Ciência Rural, v.43, p.361-365, 2013. DOI: 10.1590/S0103-84782013000200028.

TEIXEIRA, B.B.; TEIXEIRA, R.B.; SILVA, L.P. da; TORRES, R. de A.; CAETANO, G. da C.; EUCLYDES, R.F. Estimação dos componentes de variância para as características de produção e de qualidade de ovos em matrizes de codorna de corte. Ciência Rural, v.42, p.713-717, 2012. DOI: 10.1590/S0103-84782012000400023.

VENTURINI, G.C.; GROSSI, D.A.; RAMOS, S.B.; CRUZ, V.A.R.; LEDUR, M.C.; EL FARO, L.; SCHMIDT, G.S.; MUNARI, D.P. Estimation of genetic parameters for partial egg production periods by means of random regression models. Genetics and Molecular Research, v.11, p.1819-1829, 2012. DOI: $10.4238 / 2012$.July.10.18. 
WINTER, E.M.W.; ALMEIDA, M.I.M. de; OLIVEIRA, E.G. de; MARTINS, E.L.; NATEL, A.S.; SUREK, D. Aplicação do método bayesiano na estimação de correlações genéticas e fenotípicas de peso em codornas de corte em várias idades. Revista Brasileira de Zootecnia, v.35, p.1684-1690, 2006. DOI: 10.1590/S151635982006000600015 .

$\overline{\text { Recebido em } 26 \text { de dezembro de } 2012 \text { e aprovado em } 29 \text { de abril de } 2013}$ 